\title{
SREBP-1c Gene Silencing can Decrease Lipid Deposits in Bovine Hepatocytes Cultured in Vitro
}

\author{
Qinghua Denga,e Xinwei Lia ${ }^{a, e}$ Shixin Fu ${ }^{b}$ Liheng Yin ${ }^{a}$ Yuming Zhang \\ Tingting Wang ${ }^{a}$ Jianguo Wang ${ }^{c}$ Lei Liu ${ }^{a}$ Xue Yuand Guoquan Sun ${ }^{d}$ Zhe Wanga \\ Guowen Liu ${ }^{\mathrm{a}}$ Xiaobing $\mathrm{Li}^{\mathrm{a}}$ \\ aKey Laboratory of Zoonosis, Ministry of Education, College of Veterinary Medicine, Jilin University, \\ Changchun, 'DInstitute of Animal Science and Technology, Heilongjiang Bayi Agriculture University, \\ Daqing, 'College of Veterinary Medicine, Northwest A\&F University, Yangling, 'College of Animal \\ Science and Technology, Inner Mongolia National University, Tongliao, China; ${ }^{~} Q$ Qinghua Deng and \\ Xinwei Li contributed equally to this study
}

\section{Key Words}

Hepatocyte - SREBP-1c - Fat deposit

\begin{abstract}
Background: Fatty liver is a major metabolic disorder that occurs during early lactation in high-producing dairy cows. Sterol regulatory element-binding protein-1c (SREBP-1c) is an important transcription factor that regulates lipid synthesis by regulating the expression of lipid metabolism genes. Methods: In this study, we reduced the expression of SREBP-1c by adenovirus-mediated SREBP-1c with a low expression vector (AD-GFP-SREBP-1c) to study the effects of SREBP-1c on lipid deposits in bovine hepatocytes. The expression levels and enzyme activities of SERBP-1c and its target genes were determined by real-time PCR, western blot, and ELISA. Results: These results showed that Ad-GFP-SREBP-1c could inhibit SREBP-1c expression. The expression of the lipid synthesis enzyme acetyl-CoA carboxylase (ACC) was down-regulated. The expression levels of the lipid oxidation enzymes long-chain fatty acylCOA synthetase (ACSL-1), carnitine palmitoyltransferase I (CPT-I), carnitine palmitoyltransferase $\Pi(\mathrm{CPT}-\Pi)$, and $\beta$-hydroxyacyl-CoA-DH (HADH) were significantly elevated. Furthermore, the expression levels of factors involved in the assembly and transport of very low-density lipoproteins (VLDLs), such as apolipoprotein B100 (ApoB), apolipoprotein E (ApoE), and microsomal triglyceride transfer protein (MTTP) were decreased comparison with the negative control and the blank control groups, but the low-density lipoprotein receptor (LDLR) was elevated. The concentrations of TG (triglyceride) and VLDL were also reduced. Conclusion: These data suggest that low SREBP-1c expression can decrease lipid synthesis, increase lipid oxidation, and decrease the TG and VLDL content in bovine hepatocytes.
\end{abstract}

Copyright (C) 2014 S. Karger AG, Basel

Guowen Liu and Xiaobing Li 


\section{Introduction}

Fatty liver is a common multiple nutritional metabolic disease in dairy cows. The natural incidence of fatty liver is significantly higher in ruminant animals than in monogastric animals, which is explained by their special metabolic characteristics. During the early lactation period, most dairy cows experience a negative energy balance (NEB) caused by the increased demand for energy to support milk production [1]. This NEB initiates fat mobilization and a subsequent increase in the blood concentration of non-esterified fatty acid (NEFA). However, only $20 \%$ of the NEFA is used by the mammary tissue and most is absorbed by the liver. Lipid homeostasis is regulated by a family of membrane-bound transcription factors in hepatocytes. These transcription factors are designated as sterol regulatory element-binding proteins (SREBPs) and represented by three isoforms, namely SREBP-1a, SREBP-1c, and SREBP-2. SREBP-1 preferentially regulates genes involved in fatty acid and TG synthesis, and SREBP-2 primarily activates genes involved in cholesterol biosynthesis. In most animal tissues, SREBP-1c is the predominant subtype of SREBP, and it is highly expressed in adipose tissue and liver. SREBP-1c is an important nuclear transcription factor that regulates the expression of lipid metabolism genes in the liver, and it is associated with the development of fatty liver [2-4]. Studies have shown that SREBP-1c overexpression may lead to lipid metabolism disorder and cause lipid accumulation and fatty liver. However, there are many studies on over or low expression of SREBP-1c in rats and humans, while there have been few studies conducted in dairy cows [5, 6]. Studies have shown that the fat content in the liver decreased when SREBP-1c is knocked out in ob/ob mice [7]. However, the hepatic lipid metabolism in dairy cows is different from that of monogastric animals such as humans and mice. Therefore, the regulatory mechanism of SREBP-1c in relation to the incidence of fatty liver in dairy cows may be different from that of humans and mice. Therefore, we performed several related studies on cows. Bovine hepatocytes were cultured and we reduced the expression of SREBP-1c to evaluate the roles of SREBP-1c in the fatty acid metabolism of cows.

\section{Material and Methods}

\section{Cell culture and shRNA transfection}

The study protocol was approved by the Ethics Committee on the Use and Care of Animals of Jilin University (Changchun, China). The caudate lobe of the liver was obtained through liver excision by surgery on a newborn calf. Hepatocytes were isolated by a modified two-step collagenase perfusion method established by our laboratory [8]. The hepatocytes density was adjusted to $2 \times 10^{6}$ cells $/ \mathrm{mL}$ with adherent culture medium. The hepatocytes were seeded into a 6 -well tissue culture plate $(2 \mathrm{~mL}$ per well) and incubated at $37^{\circ} \mathrm{C}$ in $5 \% \mathrm{CO}_{2}$. The medium was replaced with fresh medium every $24 \mathrm{~h}$. The hepatocytes demonstrated optimum activity after being cultured for $72 \mathrm{~h}$. The cells were assigned to three groups as follows: a control group (non-transfected, $n=6$ ), a negative control group (transfected with NshRNA, $n=6$ ), and four shRNA plasmids groups (transfected with shRNA1, shRNA2, shRNA3, shRNA4 respectively, n=6).

We used GenePharma software to design the shRNA sequences, which are shown in Table 1. Four plasmids and negative controls were fluorescently labeled with FAM. The plasmids were separately transfected into hepatocytes with Lipofectamine-2000 (Invitrogen, California, USA). We used a fluorescence confocal microscope to confirm the optimum transfection time, and we then chose the most effective shRNA plasmid that could clearly lower the expression of SREBP-1c by real-time PCR.

Constructing an adenovirus low expression vector

The effective plasmid which can decrease the expression of SREBP-1c was directly subcloned into the multicloning site of the pMD-18T vector (TaKaRa Biotechnology Co., Ltd., Tokyo, Japan). The gene was then digested by KpnI/BamHI restriction enzymes and subcloned into the multicloning site of a pShuttle-BasicEGFP vector. We then transfected this vector into 293 cells to amplify and collected the virus. The virus titer ultimately reached $10^{10} \mathrm{PFU} / \mathrm{ml}$. The recombinant adenoviruses (AD-GFP- SREBP-1c) were used to infect hepatocytes, and the multiplicity of infections (MOI) were 25, 50, 100, 150, and 200 [9]. A fluorescence 
confocal microscope and flow cytometer were used to ensure the best AD-GFP-SREBP-1c concentration and the optimal time for GFP expression.

\section{Quantitative real-time RT-PCR}

After $48 \mathrm{~h}$ of infection, the total hepatocytes RNA were extracted by using Trizol according to manufacturer's instructions (TaKaRa Biotechnology Co., Ltd.). The RNA was reverse transcribed into cDNA by using a reverse transcription kit (TaKaRa Biotechnology Co., Ltd.) according to the manufacturer's instructions. The gene primers were designed with Primer Express software 5.0 and the resulting primers are shown in Table 2. All primers were synthesized by Sangon (Sangon Biotech Co., Ltd., Shanghai, China). The mRNA expression levels were evaluated by quantitative real-time polymerase chain reaction (qRT-PCR) analysis with a SYBR Green QuantiTect RT-PCR Kit (TaKaRa Biotechnology Co., Ltd.). qRT-PCR was performed on a 7500 RealTime PCR System (Applied Biosystems). The mRNA expression levels were normalized to the expression of $\beta$-actin. Real-time PCR was conducted under the following conditions: an initial denaturation at $94^{\circ} \mathrm{C}$ for $2 \mathrm{~min}, 35$ cycles of amplification (denaturation at $94^{\circ} \mathrm{C}$ for $10 \mathrm{~s}$, annealing at $60^{\circ} \mathrm{C}$ for $15 \mathrm{~s}$, and extension at $72^{\circ} \mathrm{C}$ for $30 \mathrm{~s}$ ), and extension at $72^{\circ} \mathrm{C}$ for $5 \mathrm{~min}$.

Protein extraction and western blot analysis

After $48 \mathrm{~h}$ of infection, the hepatocytes were collected and lysed in lysis buffer $(50 \mathrm{mM}$ Tris- $\mathrm{HCl}$ pH 8.0, $0.15 \mathrm{mM} \mathrm{NaCl}, 1 \%$ Triton X-100 and 100 $\mu \mathrm{M}$ PMSF), and the protein concentrations of the lysates were measured with a BCA protein assay kit (Pierce, Rockford, US). SREBP-1c, ACC and $\beta$-actin proteins were detected. For the western blot analysis, $40 \mu \mathrm{g}$ of total protein lysate was heated to $95^{\circ} \mathrm{C}$ with $4 \times$ sample buffer for $5 \mathrm{~min}$ and then resolved by $10 \%$ SDS-PAGE, and then the proteins were transferred onto PVDF membranes. After blocking, the membranes were incubated by using the $\beta$-actin, SREBP-1c and ACC antibodies (Santa Cruz Biotechnology, Santa Cruz, CA, USA), and then
Table 1. shRNA sequences and negative control (NshRNA) for SREBP-1c

\begin{tabular}{ll}
\hline Segments & Sequences $\left(5^{\prime}-3^{\prime}\right)$ \\
\hline NshRNA & GTTCTCCGAACGTGTCACGT \\
shRNA1 & GCTCTTCCATCAATGACAAGA \\
shRNA2 & GCTCTTCTCCTTGGCACTTCT \\
shRNA3 & GGACAAGGAGTTCTCAGATGC \\
shRNA4 & GCGGAGGGTATTCCTACATGA \\
\hline
\end{tabular}

Table 2. List of primers used in this paper

\begin{tabular}{ll}
\hline Genes & Primers used for PCR (5' - 3') \\
\hline SREBP-1c & F:CGACACCACCAGCATCAACCACG \\
& R:GCAGCCCATTCATCAGCCAGACC \\
3-actin & F:GCTAACAGTCCGCCTAGAAGCA \\
& R:GTCATCACCATCGGCAATGAG \\
ACC & F:TGCTGAATATCCTCACGGAGCT \\
& R:CGACGTTTCGGACAAGATGAGT \\
ACSL-1 & F:TCGGAACTGAAGCCATCACC \\
& R:GCCTCGTTCCAGCAGATCAC \\
CPT- I & F: ACGCCGTGAAGTATAACCCT \\
& R: CCAAAAATCGCTTGTCCCTT \\
CPT- I & F: TGAACATCCTCTCCATCTGG \\
& R: GGTCAACAGCAACTACTACG \\
HADH & F: GGTCCACAGCACAGACTTGGT \\
& R: GGAATTGGCTAGGCTTGTGATC \\
ApoB & F: GATACTCAGAACGGAGCAAT \\
& R: GCACCAATCAGATAACAGGA \\
ApoE & F: TCCTGAATGACCTGGGTGTTG \\
& R: TCTGTGGGTTGCCGTGGTG \\
& F: CAGTTTGCAGCCTTGGTTCTG \\
& R: ACTTTCTCCCCTGACCCTT \\
& \\
&
\end{tabular}
incubated with appropriate peroxidase-conjugated secondary antibodies (Protein technology, Chicago, USA), the resulting bands were detected using an ECL kit (Bioer, Hangzhou, China).

Detecting enzymatic activity

After infection for $48 \mathrm{~h}$, the total proteins in the hepatocytes were extracted using a biochemical kit (Gen Med Scientifics, USA). Then, we detected these samples according to the manufacturer's instructions. These samples were measured in at least three independent experiments.

Detecting the TG and VLDL contents

Hepatocytes and culture medium were collected after infection for 48 h. The cells were broken by an ultrasonic processor to detect the TG. The TG content was detected using a triglyceride detection kit 
Fig. 1. SREBP-1c gene expression levels at $24 \mathrm{~h}$ and $48 \mathrm{~h}$. TC (transfection reagent control), NC (negative control, the empty plasmid which has no shRNA of SREBP1c), control (no transfected). The values are the means $\pm S E(n=6)$. Compared with TC, NC and the control, shRNA1 and shRNA2 expression decreased $\left({ }^{* *} p<0.01\right)$. The shRNA3 and shRNA4 was * $p<0.05$.
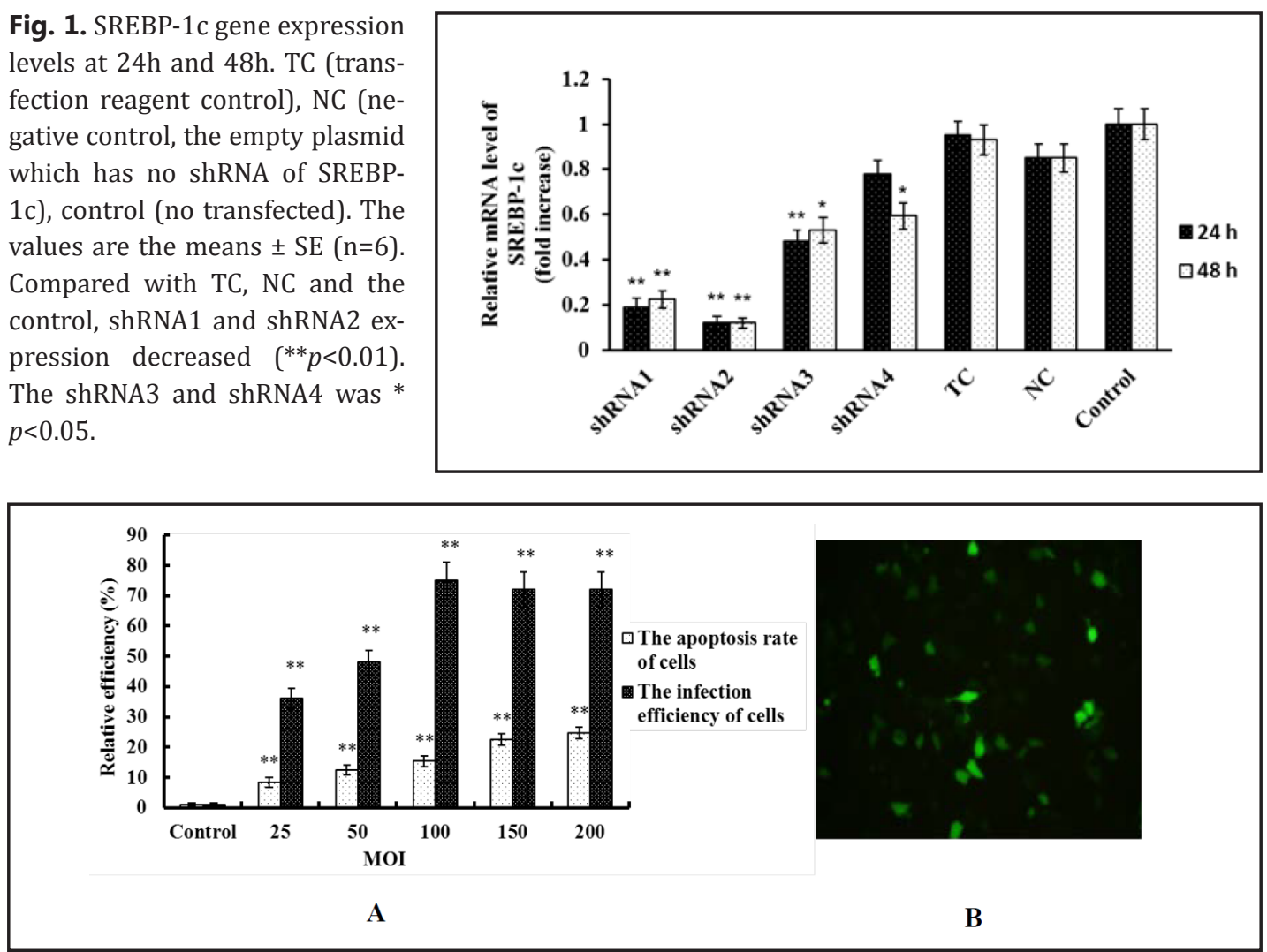

Fig. 2. A: Fluorescence confocal microscopy of hepatocytes. B: The infection efficiency and apoptosis rate of cells in different multiplicity of infections (MOI). Control (no infection). 25, 50, 100, 150, 200 (different MOIs). Values are the means \pm SE $(n=6)$. Compared with the control, the MOIs of 25, 50, 100, 150 and 200 were increased $(* * p<0.01)$.

(Jiancheng, Nanjing, China). The VLDL content of the culture medium was detected using a VLDL detection kit (Blue Gene, Shanghai, China).

\section{Statistics}

All results are expressed as the means \pm standard error (SE). Statistical analysis was performed using the statistical analysis program SPSS 13.0 (SSPS, Chicago, IL, USA). $p<0.05$ was considered significant.

\section{Results}

\section{Selecting the four plasmids}

Four plasmids (shRNA1, shRNA2, shRNA3, shRNA4) were transfected into the hepatocytes respectively. The results showed that green fluorescence was strongest at $48 \mathrm{~h}$, as observed by fluorescence confocal microscopy. The SREBP-1c expression was significantly lower in the shRNA2 and shRNA1 treatment groups than in the control groups, and the shRNA2 was the lowest (Fig. $1 ; p<0.05$ ), so we used shRNA2 to continue our studies.

\section{The optimum concentration of AD-GFP-SREBP-1c for infection}

This study confirmed the optimum concentration of AD-GFP-SREBP-1c for infection by fluorescence confocal microscopy and flow cytometry. The infection efficiency of the hepatocytes was higher, and the apoptotic rate was lower when the MOI was 100; therefore, we ensured that all the samples were collected when the MOI was 100 (Fig. $2 ; p<0.05$ ). 


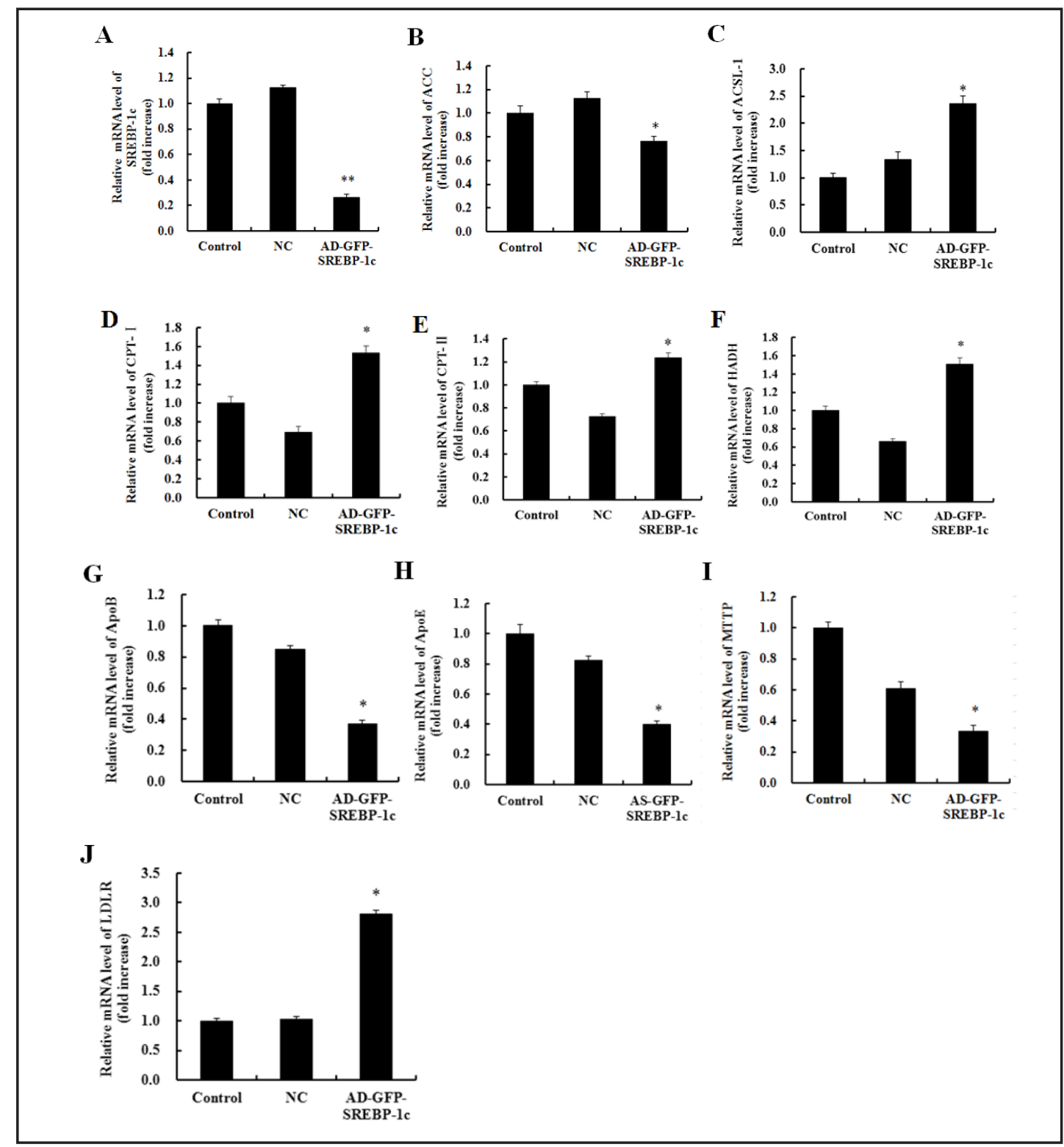

Fig. 3. The relative mRNA levels of SREBP-1c, ACC, ACSL-1, CPT- I , CPT- II , HADH, ApoB, ApoE, MTTP, and LDLR were examined by qRT-PCR after infecting AD-GFP-SREBP-1c for $48 \mathrm{~h}$. Control (no treatment), NC (negative control, empty adenovirus vector which has no SREBP-1c), AD-GFP-SREBP-1c (infected with the low expression adenovirus vector). A: The relative mRNA level of SREBP-1c was examined by qRT-PCR in hepatocytes after infecting AD-GFP-SREBP-1c for $48 \mathrm{~h}$. The values are the means $\pm \mathrm{SE}(\mathrm{n}=6)$. Compared with the control, the SREBP-1c expression decreased $\left({ }^{* *} p<0.01\right)$. B-J: The relative mRNA levels of ACC, ACSL- 1 , CPTI , CPT- II , HADH, ApoB, ApoE, MTTP, and LDLR were examined by qRT-PCR in bovine primary hepatocytes after decreasing SREBP-1c expression. Values are the means \pm SE $(n=6)$. Compared with the control, the expression of these genes clearly changed $\left({ }^{* *} p<0.01\right)$.

\section{Low SREBP-1c expression in bovine hepatocytes}

The mRNA and protein expression levels of SREBP-1c were significantly lower in the ADGFP-SREBP-1c treated hepatocytes than in the negative and control groups, which indicated that AD-GFP-SREBP-1c could inhibit the expression of SREBP-1c in bovine hepatocytes (Fig. 3A, Fig. $4 \mathrm{~B} ; p<0.05)$. 
Fig. 4. The expression levels of SREBP-1c (Fig. 4B), ACC (Fig. 4C), and $\beta$-actin (Fig. 4A) protein according to a western blot after infecting AD-GFP-SREBP-1c. Control (no infected), NC (negative control, empty adenovirus vector which has no SREBP-1c), AD-GFPSREBP-1c (infected with the low expression adenovirus vector).
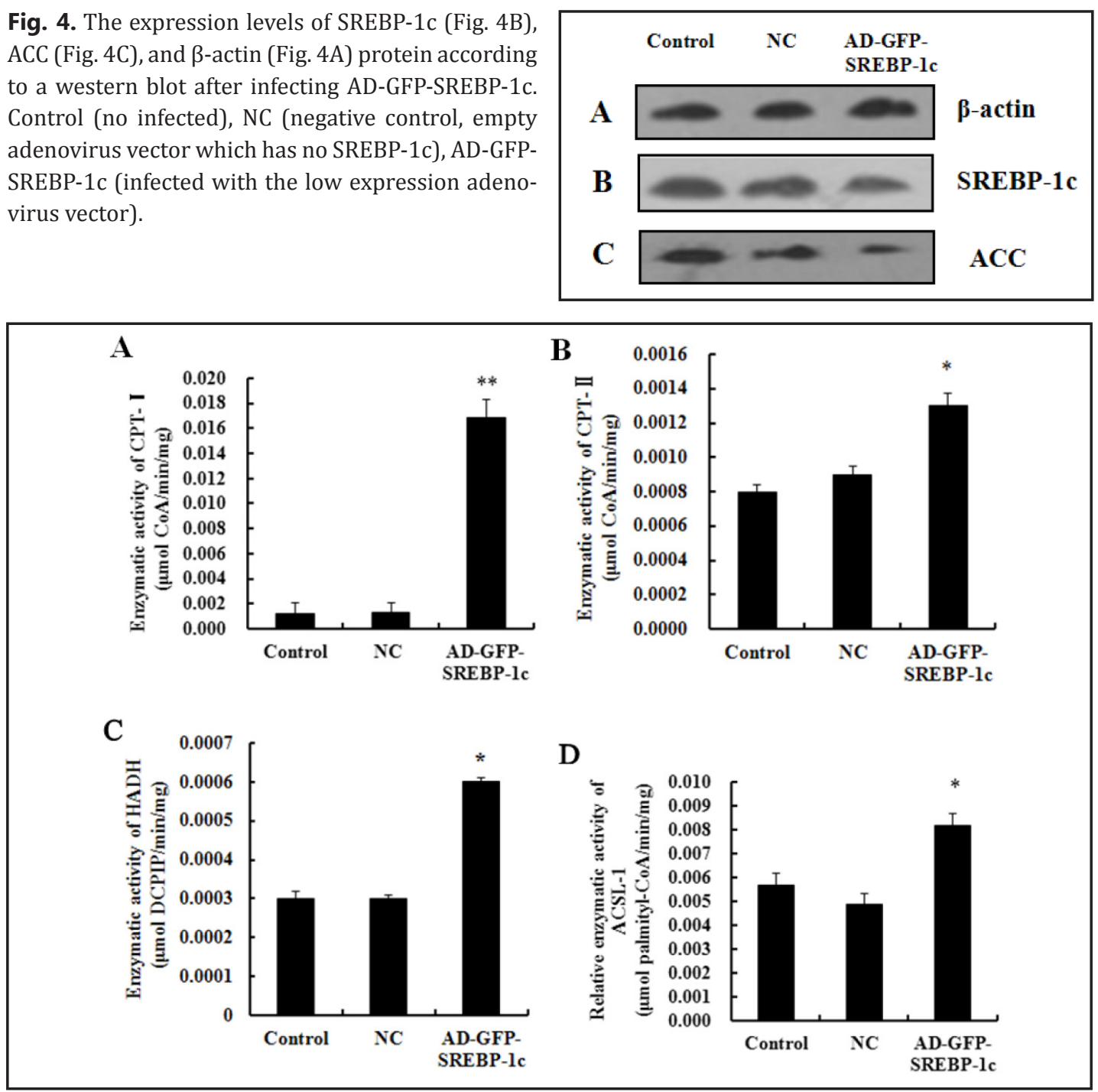

Fig. 5. The enzymatic activity results for CPT- I (Fig. 5A), CPT- II (Fig. 5B), HADH (Fig. 5C), and ACSL-1 (Fig. 5D) were examined by ELISA. Control (no infected), NC (negative control, empty adenovirus vector which has no SREBP-1c), AD-GFP-SREBP-1c (infected with the low expression adenovirus vector). The values are the means $\pm \mathrm{SE}(\mathrm{n}=3)$. Compared with the control, the enzymatic activities have clearly changed $\left({ }^{* *} p<0.01\right.$ or* $p<0.05)$.

The effect of low SREBP-1c expression on ACC expression

The mRNA and protein expression levels for ACC were significantly decreased in the bovine hepatocytes that were treated with AD-GFP-SREBP-1c (Fig. 3B, Fig. 4C; $p<0.05$ ).

The effects of low SREBP-1c expression on the expression and enzymatic activity of fatty acid oxidation enzymes

The mRNA expression levels of ACSL-1, CPT-I, CPT-П, and HADH increased significantly in bovine hepatocytes after being treated with AD-GFP-SREBP-1c (Fig. 3C, D, E, F; $p<0.05$ ). We detected the enzymatic activities after infecting with AD-GFP-SREBP-1c, and the enzymatic activities of CPT-I, CPT-П, ACSL-1 and HADH increased significantly (Fig. 5A, B, C, D; $p<0.05$ ).

The effects of low SREBP-1c expression on the assembly and secretion of VLDL

The mRNA expression levels of ApoB, ApoE, and MTTP were significantly lower in the AD-GFP-SREBP-1c treatment groups than in the control and negative control groups (Fig. 


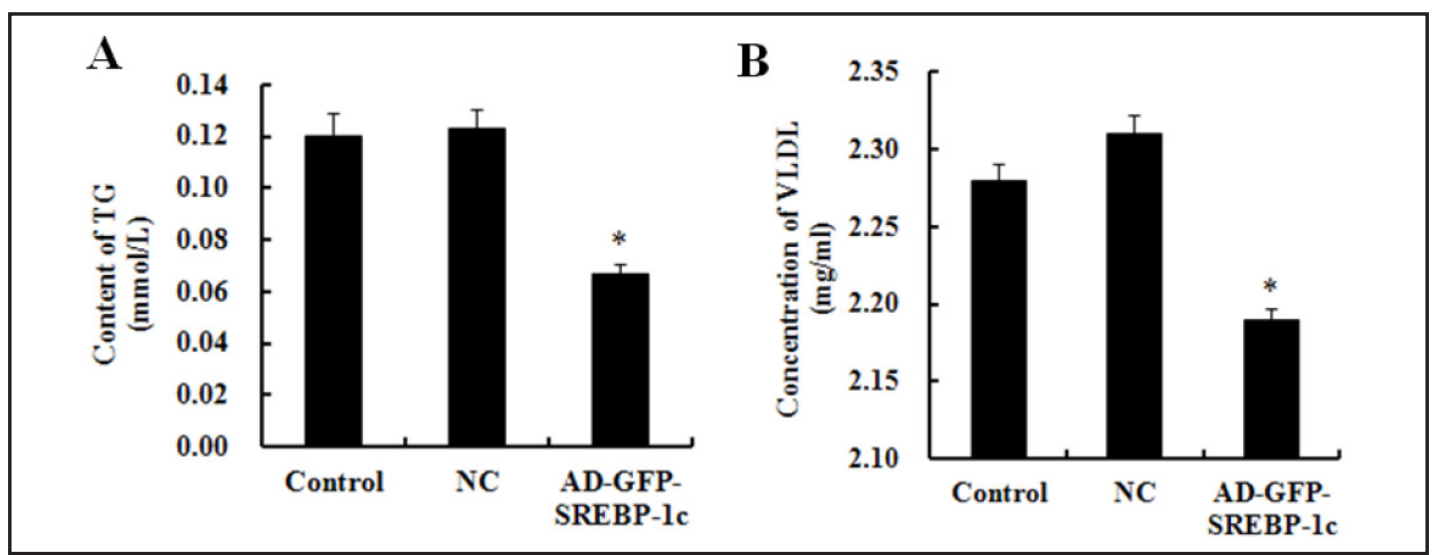

Fig. 6. The TG concentration in hepatocytes and the VLDL content in the culture medium after infecting with AD-GFP-SREBP-1c. Control (no infected), NC (negative control, empty adenovirus vector which has no SREBP-1c), AD-GFP-SREBP-1c (infected with the low expression adenovirus vector). The values are the means \pm SE $(n=3)$. Compared with the control, the TG concentration in hepatocytes and the VLDL in the liquid supernatant decreased $(* p<0.05)$.

3G, H, I; $p<0.05$ ). In addition, the mRNA expression of LDLR was significantly higher in the AD-GFP-SREBP-1c treatment groups than in the control and negative control groups (Fig. 3J; $p<0.05$ ).

\section{TG and VLDL contents}

To confirm the effects of low SREBP-1c expression on the fatty acid metabolism in bovine hepatocytes, we detected the TG contents in hepatocytes and VLDL in the culture medium. The TG content was significantly lower in the AD-GFP-SREBP-1c treatment groups than in the control groups (Fig. 6A; $p<0.05$ ), and the VLDL content was also significantly lower than that of the control groups (Fig. 6B; $p<0.05$ ).

\section{Discussion}

Fatty liver occurs in dairy cows during periods of elevated blood NEFA. Elevated blood NEFA levels are associated with NEB and hormonal changes at parturition. Overfeeding during the dry period leads to a deposition of body fat, and the dry matter intake (DMI) is beyond repair during the short postpartum period. The degree of lipid mobilization and NEB is worse, consequently, the incidence of fatty liver in overweight cows is higher. Fatty liver is a nutritional and metabolic disease, and it primarily occurs in high yield cows during the perinatal period, it is a high incidence disease and has an enormous influence on cows' health and productivity.

SREBP-1c is a subtype of SREBPs, and it is a key transcription factor that regulates intracellular lipid metabolism. Some studies have shown that SREBP-1c regulated the expression of lipid synthesis genes and then influenced hepatic lipid metabolism [10]. Studies have indicated that the overexpression of SREBP-1c could lead to a disorder in lipid metabolism, and then cause fat accumulation in non-adipose tissue such as the liver [11]. Fatty liver is characterized by insulin resistance (IR) and is associated with SREBP-1c. The liver volume increased 33\% in rats with SREBP-1c over-expression, and the TG also clearly increased and leading to fatty liver. When SREBP-1c was overexpressed in transgenic mouse livers, fatty acid synthesis increased 26 times, and the synthesis of cholesterol increased 5 times [12]. The hepatic lipid metabolism of dairy cows is different from that of monogastric animals such as humans and mice. The regulation of SREBP-1c in the hepatic lipid metabolism of dairy cows may also be different [13]. Therefore, bovine hepatocytes were cultured and 
treated with AD-GFP-SREBP-1c to reduce the expression of SREBP-1c, thereby indicating the effects of SREBP-1c on hepatic lipid metabolism.

ACC is the most important enzyme during fatty acid synthesis [14], and it is enriched in the liver, adipose tissue, and lactating mammary gland, where it catalyzes the biosynthesis of long-chain fatty acids [15]. At the transcriptional level, ACC expression is regulated by a group of transcription factors, and SREBP-1c is the most important of these [16, 17]. ACC is regulated by SREBP-1c in the fatty acid synthesis pathway. Previous studies have shown that ACC expression was reduced when SREBP-1c was inhibited [18]. In view of the references discussed above, we can infer that elevating ACC expression could increase fat synthesis and then induce fat deposition in the liver, and it may cause fatty liver in cows and NAFLD (NonAlcoholic Fatty Liver Disease) in humans. However, the mRNA and protein expression levels of ACC in this study were significantly decreased after decreasing the expression of SREBP1c. This finding indicates that low SREBP-1c expression can decrease fatty acid synthesis in bovine hepatocytes. However, a large quantity of fat will accumulate when the bovine develops fatty liver, which may be caused by increased fat synthesis or/and decreased lipid oxidation. ACC is the key gene in the fatty acid synthesis process, and the ACC level may markedly increase when cows have fatty liver. In our study, we found that lowering SREBP1c expression can reduce the mRNA and protein levels of ACC, and we can infer that ACC can lower fatty acid synthesis and reduce the incidence of fatty liver in cows if there is no lipid oxidation effect. Humans and mice are the same in that fat accumulation can result in NAFLD [19], and decreasing the expression of ACC may reduce the incidence of NAFLD in mice and humans. We can speculate that lowering the expression of SREBP-1c may treat fatty liver in cows, and this discovery provides a foundation for gene-based therapies for fatty liver.

The fatty acid oxidation process includes the activation of fatty acids, in which the fatty acid enters mitochondria and undergoes $\beta$-oxidation. During the $\beta$-oxidation of a fatty acid, TG is hydrolyzed into FFA and glycerine by lipase, and FFA generates energy by $\beta$-oxidation, however, the fatty acid catabolism process is completed by ACSL-1 [20]. During this process, the fatty acid enters the mitochondria. The key enzymes are CPT-I and CPT-П [21,22]. CPT-I can transport activated fatty acids, and the integral outer membrane protein converts activated fatty acids into acylcarnitines [23]; СРТ-П is the key enzyme for fatty acid transport and it is located in the mitochondrial inner membrane [24]. CPT-I expression was reduced in NAFLD patients, and increasing the expression of CPT-I can lower the accumulation of TG. However, the TG level in the liver clearly increased when the CPT-I activity was decreased [25]. The process of fatty acid $\beta$-oxidation also includes key enzymes HADH. HADH catalyzes the third reaction of the mitochondrial $\beta$-oxidation cascade, namely the oxidation of 3-hydroxyacylCoA to 3-ketoacyl-CoA [26]. We found that the expression levels of the above genes increased to different degrees, which indicated that low SREBP-1c expression could promote lipid oxidation in the hepatocytes. If fatty acids that were disaggregated and accumulated in the liver were decreased, the rate of fatty liver formation would decrease. In addition, we found that the enzyme activities of the above genes were elevated. This finding demonstrates that low SREBP-1c expression increased the enzyme activity of lipid oxidation genes. The increased expression levels of lipid oxidation genes could reduce the ratio of lipogenesis and lipid oxidation, and then indirectly reduce lipid deposition in the liver. NAFLD in humans and fatty liver in cows result from the accumulation of a large amount of lipids in the liver, leading to an increase ratio of lipogenesis and lipid oxidation. We found that a decrease in the SREBP-1c expression level largely enhanced the expression level of lipid oxidation genes and the activity of the key enzymes in the lipid oxidation process. In addition, the ratio of lipogenesis and lipid oxidation may diminish if the level of lipogenesis does not change. We can then infer that lowering the expression level of SREBP-1c may reduce the amount of fat that accumulates in the livers of humans and cows, and it may reduce the morbidity of fatty liver in cows and NAFLD in humans.

Key factors in the assembly of VLDL (very low density lipoprotein) include ApoB, ApoE, MTTP, and LDLR. TG exported in the form of VLDL requires coordinated functions of ApoB and MTTP $[27,28]$. ApoB and ApoE are important proteins for the assembly and secretion of 
VLDL $[29,30]$. MTTP is very important in the early stage of assembly of ApoB, and the MTTP combines with $A$ poB in the endoplasmic reticulum as a carrier, and is then transported outside the liver as a very low density lipoprotein [31-33]. MTTP expression decreased and was consistent with the change in ApoB. The TG and VLDL contents and the expression levels of ApoB, ApoE, MTTP and LDLR were detected. We found that the expression levels of ApoB, ApoE, and MTTP decreased, and LDLR was significantly elevated in the hepatocytes after decreasing the SREBP-1c expression. The elevated expression of LDLR could inhibit the ApoB secretion and regulate the assembly of VLDL by blocking the secretion of VLDL [34]. The accumulation of TG in the liver is the most direct cause of fatty liver. However, the most important reason for TG accumulation is increased fatty synthesis and reduced transfer out of the liver or reduced TG hydrolysis. VLDL is the most important form of transport TG, and the TG concentration may affect the VLDL content. In this study, the TG concentration in the hepatocytes was markedly reduced when the SREBP-1c expression was decreased, which may have been caused by the low expression of ApoB, ApoE, and MTTP. A reduction in the VLDL can decrease the TG accumulation and then potentially decrease the incidence of fatty liver.

In conclusion, low SREBP-1c expression decreased lipid synthesis and VLDL assembly, and increased lipid oxidation, thereby decreasing the TG content of hepatocytes.

\section{Abbreviations}

SREBP-1c (sterol regulatory element binding protein-1c); VLDL (very low density lipoprotein); CPT-I (carnitine palmityl transferase I); CPT- II (carnitine palmityl transferase II ); ACC (acetyl-CoA carboxylase); ACSL-1 (long-chain fatty acyl-COA synthetase 1); HADH ( $\beta$-hydroxyacyl-CoA-DH); ApoB100 (apolipoprotein B100); ApoE (apolipoprotein E); MTTP (microsomal triglyceride transfer protein); LDLR (low-density lipoprotein receptor); NEB (hepatic lipidosis in negative energy balance); NEFA (non-esterified fatty acids); TG (triglyceride); MOI (Multiplicity of infections); DMI (dry matter intake).

\section{Acknowledgments}

This work was supported by the Program for New Century Excellent Talents in University (NCET-11-0199), the National Key Technology R\&D Program (grant no. 2012BAD12B03), and the National Natural Science Foundation of China (Beijing, China; grant numbers: 30871897, 30972224, 31072178, 31172372, 31172172, 31201961, and 31272621).

\section{References}

1 Li X, Chen H, Lei L, Liu J, Guan Y, Liu Z, Zhang L, Yang W, Zhao C, Fu S, Li P, Liu G, Wang Z: Non-esterified fatty acids activate the amp-activated protein kinase signaling pathway to regulate lipid metabolism in bovine hepatocytes. Cell Biochem Biophys 2013;67:1157-1169.

-2 Ahmed MH, Byrne CD: Modulation of sterol regulatory element binding proteins (srebps) as potential treatments for non-alcoholic fatty liver disease (nafld). Drug Discov Today 2007;12:740-747.

-3 Goldstein JL, DeBose-Boyd RA, Brown MS: Protein sensors for membrane sterols. Cell 2006;124:35-46.

4 Horton JD, Goldstein JL, Brown MS: Srebps: Activators of the complete program of cholesterol and fatty acid synthesis in the liver. J Clin Invest 2002;109:1125-1131.

5 Nasr Z, Dow LE, Paquet M, Chu J, Ravindar K, Somaiah R, Deslongchamps P, Porco Jr JA, Lowe SW, Pelletier J: Suppression of eukaryotic initiation factor $4 \mathrm{e}$ prevents chemotherapy-induced alopecia. BMC Pharmacol Toxicol 2013;14:58.

6 Boivin B, Chaudhary F, Dickinson BC, Haque A, Pero SC, Chang CJ, Tonks NK: Receptor protein-tyrosine phosphatase alpha regulates focal adhesion kinase phosphorylation and erbb2 oncoprotein-mediated mammary epithelial cell motility. J Biol Chem 2013;288:36926-36935. 
7 Yahagi N, Shimano H, Hasty AH, Matsuzaka T, Ide T, Yoshikawa T, Amemiya-Kudo M, Tomita S, Okazaki H, Tamura Y, Iizuka Y, Ohashi K, Osuga J, Harada K, Gotoda T, Nagai R, Ishibashi S, Yamada N: Absence of sterol regulatory element-binding protein-1 (srebp-1) ameliorates fatty livers but not obesity or insulin resistance in lep(ob)/lep(ob) mice. J Biol Chem 2002;277:19353-19357.

8 Li X, Bai G, Chen H, Deng Q, Liu Z, Zhang L, Liu G, Wang Z: Effects of non-esterified fatty acids on the gluconeogenesis in bovine hepatocytes. Mol Cell Biochem 2012;359:385-388.

-9 Fu SX, Deng QH, Yang WT, Ding HY, Wang XX, Li P, Li XW, Wang Z, Li XB, Liu GW: Increase of fatty acid oxidation and vldl assembly and secretion overexpression of pten in cultured hepatocytes of newborn calf. Cell Physiol Biochem 2012;30:1005-1013.

-10 Ferre P, Foufelle F: Srebp-1c transcription factor and lipid homeostasis: Clinical perspective. Horm Res 2007;68:72-82.

11 Li Y, Zhang Y, Li R, Chen W, Howell M, Zhang R, Chen GX: The hepatic raldh1 expression is elevated in zucker fatty rats and its over-expression introduced the retinal-induced srebp-1c expression in ins- 1 cells. Plos One 2012;7

12 Kim JB, Sarraf P, Wright M, Yao KM, Mueller E, Solanes G, Lowell BB, Spiegelman BM: Nutritional and insulin regulation of fatty acid synthetase and leptin gene expression through add1/srebp1. J Clin Invest 1998;101:1-9.

13 Parvy JP, Napal L, Rubin T, Poidevin M, Perrin L, Wicker-Thomas C, Montagne J: Drosophila melanogaster acetyl-coa-carboxylase sustains a fatty acid-dependent remote signal to waterproof the respiratory system. PLoS Genet 2012;8:e1002925.

14 Choi S, Choi Y, Choi Y, Kim S, Jang J, Park T: Piperine reverses high fat diet-induced hepatic steatosis and insulin resistance in mice. Food Chem 2013;141:3627-3635.

15 Liu L, Yang M, Lin X, Li Y, Liu C, Yang Y, Yamahara J, Wang J: Modulation of hepatic sterol regulatory element-binding protein-1c-mediated gene expression contributes to salacia oblonga root-elicited improvement of fructose-induced fatty liver in rats. J Ethnopharmacol 2013;150:1045-1052.

-16 Foufelle F, Ferre P: New perspectives in the regulation of hepatic glycolytic and lipogenic genes by insulin and glucose: A role for the transcription factor sterol regulatory element binding protein-1c. Biochem J 2002;366:377-391.

17 Eberle D, Hegarty B, Bossard P, Ferre P, Foufelle F: Srebp transcription factors: Master regulators of lipid homeostasis. Biochimie 2004;86:839-848.

18 Suchankova G, Tekle M, Saha AK, Ruderman NB, Clarke SD, Gettys TW: Dietary polyunsaturated fatty acids enhance hepatic amp-activated protein kinase activity in rats. Biochem Biophys Res Commun 2005;326:851-858.

19 Boden G, Salehi S, Cheung P, Homko C, Song W, Loveland-Jones C, Jayarajan S: Comparison of in vivo effects of insulin on srebp-1c activation and insig-1/2 in rat liver and human and rat adipose tissue. Obesity (Silver Spring) 2013;21:1208-1214.

20 Liu T, Yang LL, Zou L, Li DF, Wen HZ, Zheng PY, Xing LJ, Song HY, Tang XD, Ji G: Chinese medicine formula lingguizhugan decoction improves beta-oxidation and metabolism of fatty acid in high-fat-diet-induced rat model of fatty liver disease. Evid Based Complement Alternat Med 2013;2013:429738.

-21 Linher-Melville K, Zantinge S, Sanli T, Gerstein H, Tsakiridis T, Singh G: Establishing a relationship between prolactin and altered fatty acid beta-oxidation via carnitine palmitoyl transferase 1 in breast cancer cells. BMC Cancer 2011;11:56.

22 Al-Aqeel AI, Rashed MS, Ruiter JP, Al-Husseini HF, Al-Amoudi MS, Wanders RJ: Carnitine palmityl transferase i deficiency. Saudi Med J 2001;22:1025-1029.

23 Lee K, Kerner J, Hoppel CL: Mitochondrial carnitine palmitoyltransferase 1a (cpt1a) is part of an outer membrane fatty acid transfer complex. Journal of Biological Chemistry 2011;286:25655-25662.

$\checkmark 24$ Dobrzyn P, Dobrzyn A, Miyazaki M, Cohen P, Asilmaz E, Hardie DG, Friedman JM, Ntambi JM: Stearoyl-coa desaturase 1 deficiency increases fatty acid oxidation by activating amp-activated protein kinase in liver. $\mathrm{P}$ Natl Acad Sci USA 2004;101:6409-6414.

25 Dobbins RL, Szczepaniak LS, Bentley B, Esser V, Myhill J, McGarry JD: Prolonged inhibition of muscle carnitine palmitoyltransferase-1 promotes intramyocellular lipid accumulation and insulin resistance in rats. Diabetes 2001;50:123-130. 


\section{Cellular Physiology $\quad$ Cell Physiol Biochem 2014;33:1568-1578 and Biochemistry \\ Deng et al.: SREBP-1c Gene Silencing Can Decrease Lipid Deposits}

-26 Schulz N, Himmelbauer H, Rath M, van Weeghel M, Houten S, Kulik W, Suhre K, Scherneck S, Vogel H, Kluge R, Wiedmer P, Joost HG, Schurmann A: Role of medium- and short-chain l-3-hydroxyacyl-coa dehydrogenase in the regulation of body weight and thermogenesis. Endocrinology 2011;152:4641-4651.

27 Meshkani R, Adeli K: Hepatic insulin resistance, metabolic syndrome and cardiovascular disease. Clin Biochem 2009;42:1331-1346.

28 Higuchi N, Kato M, Tanaka M, Miyazaki M, Takao S, Kohjima M, Kotoh K, Enjoji M, Nakamuta M, Takayanagi R: Effects of insulin resistance and hepatic lipid accumulation on hepatic mrna expression levels of apob, mtp and l-fabp in non-alcoholic fatty liver disease. Exp Ther Med 2011;2:1077-1081.

29 Shelness GS, Sellers JA: Very-low-density lipoprotein assembly and secretion. Curr Opin Lipidol 2001;12:151-157.

30 Spann NJ, Kang S, Li AC, Chen AZ, Newberry EP, Davidson NO, Hui STY, Davis RA: Coordinate transcriptional repression of liver fatty acid-binding protein and microsomal triglyceride transfer protein blocks hepatic very low density lipoprotein secretion without hepatosteatosis. Journal of Biological Chemistry 2006;281:33066-33077.

-31 Sparks JD, Sparks CE: Overindulgence and metabolic syndrome: Is foxo1 a missing link? J Clin Invest 2008;118:2012-2015.

32 Plonne D, Schulze HP, Kahlert U, Meltke K, Seidolt H, Bennett AJ, Cartwright IJ, Higgins JA, Till U, Dargel R: Postnatal development of hepatocellular apolipoprotein b assembly and secretion in the rat. J Lipid Res 2001;42:1865-1878.

-33 Taghibiglou C, Rudy D, Van Iderstine SC, Aiton A, Cavallo D, Cheung R, Adeli K: Intracellular mechanisms regulating apob-containing lipoprotein assembly and secretion in primary hamster hepatocytes. J Lipid Res 2000;41:499-513.

34 Argov N, Moallem U, Sklan D: Lipid transport in the developing bovine follicle: Messenger rna expression increases for selective uptake receptors and decreases for endocytosis receptors. Biol Reprod 2004;71:479-485. 